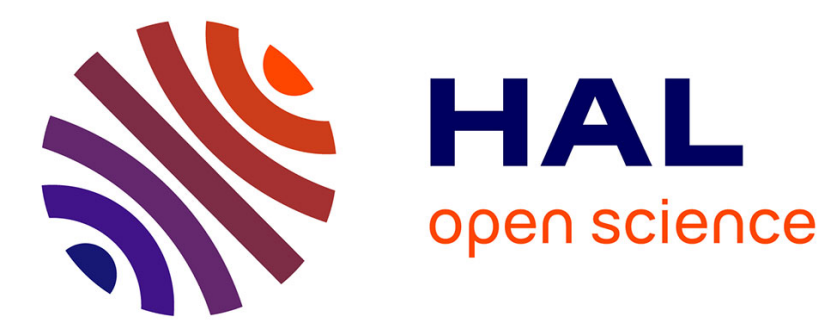

\title{
Modeling electroluminescence in insulating polymers under ac stress: effect of excitation waveform
}

\author{
Fulbert Baudoin, D H Mills, P L Lewin, Séverine Le Roy, G. Teyssedre, C
}

Laurent

\section{To cite this version:}

Fulbert Baudoin, D H Mills, P L Lewin, Séverine Le Roy, G. Teyssedre, et al.. Modeling electroluminescence in insulating polymers under ac stress: effect of excitation waveform. Journal of Physics D: Applied Physics, 2011, 44 (16), pp.165402. 10.1088/0022-3727/44/16/165402 . hal-00613272

\section{HAL Id: hal-00613272 \\ https://hal.science/hal-00613272}

Submitted on 4 Aug 2011

HAL is a multi-disciplinary open access archive for the deposit and dissemination of scientific research documents, whether they are published or not. The documents may come from teaching and research institutions in France or abroad, or from public or private research centers.
L'archive ouverte pluridisciplinaire HAL, est destinée au dépôt et à la diffusion de documents scientifiques de niveau recherche, publiés ou non, émanant des établissements d'enseignement et de recherche français ou étrangers, des laboratoires publics ou privés. 


\title{
Modeling electroluminescence in insulating polymers under $\mathrm{AC}$ stress: effect of excitation waveform
}

\author{
F Baudoin ${ }^{2}$, D H Mills ${ }^{1}$, P L Lewin ${ }^{1}$, S Le Roy ${ }^{2,3}$, G Teyssedre ${ }^{2,3}$ and C Laurent ${ }^{2,3}$ \\ ${ }^{1}$ University of Southampton, The Tony Davies High Voltage Laboratory, United \\ Kingdom \\ ${ }^{2}$ University of Toulouse, UPS, INPT, LAPLACE (Laboratoire Plasma et Conversion \\ d'Energie), France \\ 118 route de Narbonne, F-31062 Toulouse cedex 9, France. \\ ${ }^{3}$ CNRS ; LAPLACE ; F-31062 Toulouse, France. \\ E-mail: fulbert.baudoin@laplace.univ-tlse.fr
}

\begin{abstract}
A charge transport model allowing the description of electroluminescence in polyethylene films under AC stress is proposed. The fluid model incorporates bi-polar charge injection/extraction, transport and recombination. The physics is based on hopping-mobility of electronic carriers between traps with an exponential distribution in which trap-filling controls the mobility. The computation mesh is very tight close to the electrodes -of the order of $0.4 \mathrm{~nm}$ allowing mapping the density of positive and negative carriers during sinusoidal, triangular and square $50 \mathrm{~Hz}$ voltage waveforms. Experiment and simulation fit nicely and the time-dependence of the electroluminescence intensity is accounted for by the charge behaviour. Light emission scales with the injection current. It is shown that space charge affects a layer of $10 \mathrm{~nm}$ away from the electrode where the mobility is increased as compared to the bulk mobility due to the high density of charge. The approach is very encouraging and opens the way to model space charge under timevarying voltages.
\end{abstract}

PACS Numbers: $72.20 \mathrm{Ht}, 72.20 \mathrm{Jv}$ 


\section{Introduction}

Space charge in insulating polymeric materials has been the subject of a number of investigations aiming at understanding their effect on the field distribution and their interaction with the material. Most of the published work concerns the field modulation that any space charge in the material will induce [1]. Much less attempts have been done in understanding the physics of their interaction with the polymer, e. g. the nature of the trapping centres [2] or their impact in energetic terms [3]. The latter is thought to be important when it comes to understanding ageing processes: trapped charges are known to change the electromechanical energy stored locally, thereby inducing a change in the chemical reactivity of the charged centres [4]. Upon charge recombination chemically reactive excited states are created that could induce local atomic rearrangements leading to material degradation [5]. Evidence of the existence of excited states has been brought up by electroluminescence (EL) detection: in carefully-controlled experiments where light from ionisation phenomena in gas is avoided, light emission has been shown to be characteristic of the polymeric material and its degradation [5].

Observation of EL has been reported for long in divergent [6] and uniform [7] field configurations, for DC and time-varying electrical stresses [8], in different kinds of materials, but models of EL are scarce. One approach has been developed by Alison et al [9] to explain EL under AC stress in epoxy resin with a pinplane geometry [10]. The model postulates the development of a space charge region near the injecting contact, the inner sphere in a concentric sphere arrangement or a flat electrode in uniform field configuration, with a uniform distribution within a thin layer (typically $100 \mu \mathrm{m}$ ) relative to the electrode spacing (order of $1 \mathrm{~mm}$ ). The space charge is established instantaneously in the space charge volume, which remains constant over time, irrespective of the applied field, i.e. charge transport is not taken into account. Injected charges during an AC half cycle are either trapped or recombined with the trapped charges of opposite polarity injected during the previous half cycle. The model is able to reproduce the experimental data, especially in terms of the phase angle between EL and the applied voltage as well as the shape of the EL distribution within each half cycle of the applied voltage. More recently, Ariffin et al [11] have developed a model based on the same concepts but considering a smaller penetration depth (order of $10 \mathrm{~nm}$ ) of the injected charges into the dielectric. Phase angle between EL and applied stress is correctly described. Other modelling approaches of EL under AC have been attempted by Le Roy et al [12] on the basis of a transport model that has been successful in describing EL under DC field. However, by taking the model parameters satisfying the DC situation, it has not been possible to reproduce the EL phase angle observed under AC stress.

It should be noted that the models having some success in describing the EL under AC stress are those enabling the build-up of a space charge region with characteristics (density and volume) sufficient to significantly oppose the applied field. In doing so, the electric field at the injecting electrode is controlled by the space charge and can even be reversed when the applied voltage is small, e.g. when approaching the zero crossing voltage. It gives the condition for a phase shift between the applied field and the space charge-mediated field at the injecting contact, controlling injection of charges and subsequent recombination processes.

Supposing that EL emission pattern reflects the field at the injecting electrode, for explaining the phase advance of EL in respect to applied field (typically $30-40^{\circ}$ for uniform field configuration), the amount of stored charge must be such that the space charge-induced field represents $20 \%$ of the applied crest field. In previous models, the order of magnitude of the space charge density allowing field mediation at the injecting electrode in a uniform field configuration is typically $0.1 \mathrm{C} . \mathrm{m}^{-3}$ when considering a $100 \mu \mathrm{m}$ space charge layer [9]; it is of $10^{+4} \mathrm{C} \cdot \mathrm{m}^{-3}$ when considering a carrier penetration depth of $10 \mathrm{~nm}$ as in [11] with quite different applied field amplitude. Having said so, the question that is raised is the physical soundness of these models. It is known that injected charges proceed via a succession of trapping/detrapping steps within the bulk of the dielectric and they not distribute instantaneously and uniformly within the postulated space charge region.

The only way to promote a physically-sound approach is to use a transport model able to describe charge movement near the injecting surface within distances comparable to the mean free path of carriers (i.e. the distance between two trapping sites). With that in mind we introduced a model of EL under time-varying 
stresses incorporating injection, transport and extraction of charges. It has been proposed a few years ago by Boufayed et al. [13] to describe charge transport in disordered materials considering an exponential distribution in the energy of trapping levels. This description, together with the transport mechanism of thermally-activated hopping type, is a classical and well accepted description in disordered materials [14]. The paper comprises four sections. The first section includes a description of the physical model. In the second section the phase-resolved EL measurements are described. Simulation and experimental results are presented in the third section for different applied voltage waveforms. Finally, a general discussion of the results and of their implication, together with consideration of the physical meaning of the optimized set of model parameters is presented.

\section{Model description}

\subsection{Physical description}

The bipolar model developed is based on [13]. It is one-dimensional along the direction $x$ perpendicular to the plane of the dielectric film, Figure 1.

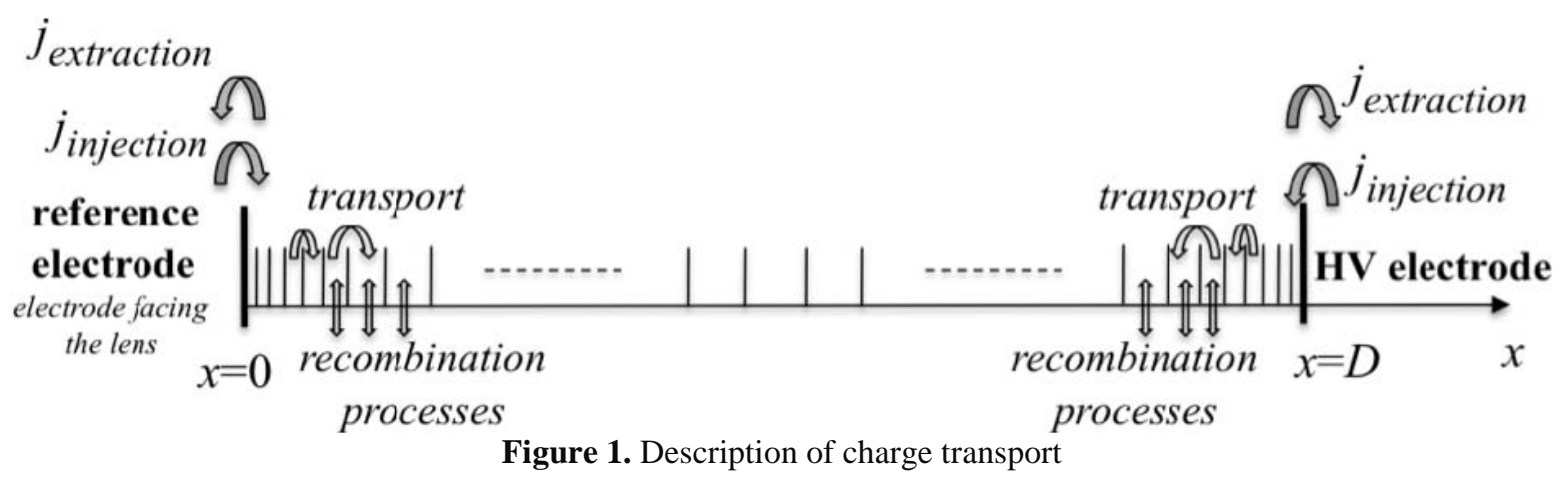

Charges are provided by injection at electrodes according to a functional approximation for the current density calculation established in [15]:

$$
j_{e, h}(x, t)=\alpha \exp (\beta E(x, t))
$$

$D$ is the dielectric thickness. Thus, $j_{e}(0, t)$ and $j_{h}(D, t)$ are the injection fluxes of electrons and holes respectively when the HV electrode is at positive polarity, $\alpha$ and $\beta$ are constants. This injection law has been preferred to the conventional Richardson-Schottky equation since the latter leads to some inconsistency regarding the barrier height value [12]. Hare et al. [15] have shown that the description holds for field values up to about $300 \mathrm{kV} \cdot \mathrm{mm}^{-1}$.

Charge transport is described by a hopping mechanism in which carriers move from site to site by getting over a potential barrier leading to a mobility expression of the form:

$$
\mu=\frac{2 v d}{E} \exp \left(-\frac{\Delta_{f}}{k T}\right) \sinh \left(\frac{e E d}{2 k T}\right)
$$

where $v$ is the attempt-to-jump frequency, $d$ is the average distance between traps, $T$ is the temperature, $e$ is the elementary charge and $\Delta_{f}$ is the upper filled level of the traps distribution. As the average inter-site distance $d$ is related to the trap density, only one extra-parameter is needed, $v$, which has been set to $k T / h$ $=6.2 \times 10^{12} \mathrm{~s}^{-1}$, where $h$ is the Planck's constant. 
The model takes into account trap filling effects by considering that $\Delta_{\mathrm{f}}$ depends on the space charge accumulation at a given position. This is done by assuming an exponential distribution of trapping sites in energy, according to:

$$
N_{t(e, h)}\left(\Delta_{e, h}\right)=N_{e, h}^{\prime} \exp \left(-\frac{\Delta_{e, h}}{k T_{0(e, h)}}\right)
$$

where $\Delta$ is the trap depth (with $\Delta \leq \Delta_{\max }$ ), $k$ is Boltzmann's constant, $N_{t(e)}$ and $N_{t(h)}$ are the trap density distribution respectively for electrons and holes characterized by a shape parameter $T_{0}$, a pre-exponential factor $N^{\prime}$ and a maximum trap depth $\Delta_{\max }$.

When $T \ll<T_{0}$, only a fraction $n_{f}$ of the total charge $n_{t}$ is involved in transport (the indexes $f$ and $t$ denote respectively the fraction of mobile charges and trapped charges):

$$
n^{f}=\frac{n^{t}}{1+a} \text { with } a=\frac{T_{0}}{T}>1
$$

Within this model, the hopping charges come from the highest filled trap state at a depth $\Delta_{f}$ and the traps are filled from the deepest available site upwards. The overall trap-filling modulated hopping mechanism is shown in Figure 2. Charges participating to the transport are those located within kT below the highest occupied levels at $\Delta_{\mathrm{f}}$.

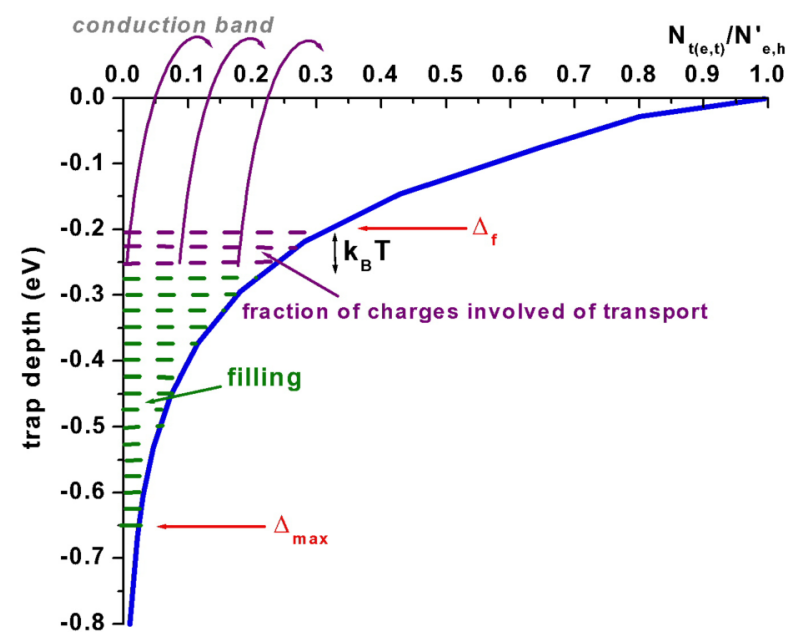

Figure 2. Trap-filling modulated hopping mechanism incorporated in the model (distribution of trap levels is shown here using parameters: $\mathrm{T}_{0}=10^{4} \mathrm{~K}, \Delta_{\max }=0.67 \mathrm{eV}$ )

Charge extraction is assumed to occur without a potential barrier (ohmic contact for extraction) and charge recombination is taken into account: charges injected or transported will either be trapped or recombine with the trapped charges of opposite polarity. The mobile-trapped charge carrier recombination density over a time interval is proportional to the density of mobile and trapped charges:

$$
r_{e}^{f}=S_{e, h} n_{e}^{f} n_{h}^{t} \text { and } r_{h}^{f}=S_{h, e} n_{h}^{f} n_{e}^{t}
$$

where $r_{e}^{f}$ is the recombination density for mobile electrons-trapped holes per unit time, $r_{h}^{f}$ is the recombination density of mobile holes-trapped electrons per unit time and $S_{e, h}$ and $S_{h, e}$ are the respective 
recombination coefficients. The recombination terms have been included in the model in the same way as in a previous model [16], considering only recombination between mobile and trapped species. The resultant space-averaged recombination density, which is the quantity to be compared to the EL intensity, can be written:

$$
I=\frac{1}{D} \int_{0}^{D}\left[r_{e}^{f}(x, t)+r_{h}^{f}(x, t)\right] d x
$$

Poisson's equation, continuity equation and transport equation are used to recover the net density of charges within the dielectric.

$$
\begin{gathered}
\frac{\partial^{2} V(x, t)}{\partial x^{2}}=\frac{n_{e}^{t}(x, t)+n_{h}^{t}(x, t)}{\mathcal{E}_{0} \varepsilon_{r}} \\
\frac{\partial n_{e, h}^{t}(x, t)}{d t}+\frac{\partial j_{e, h}(x, t)}{d x}=s(x, t) \\
j_{e, h}(x, t)=n_{e, h}^{f}(x, t) \mu_{e, h}(x, t) E(x, t)
\end{gathered}
$$

where $t$ is the time, $s=r_{e}^{f}+r_{h}^{f}$ the source term, $\varepsilon_{0} \varepsilon_{r}$ the dielectric permittivity with $\varepsilon_{r}=2.3$ for polyethylene.

\subsection{Numerical resolution}

The mesh used for computation, Figure 1, is divided into 1500 elements $\Delta x$, of varying lengths, being tightened close to the electrodes: 10 cells are used within a distance of $3.6 \mathrm{~nm}$ from the electrode. This feature is very important because as will be shown later on, charges remain very close to the interface. Therefore, tight space discretization is necessary to observe profile evolution [17]. This is understandable since charge injection and extraction will occur continuously each half cycle so charge carriers will have a limited penetration depth. Using this mesh, the time step $\Delta t$, has to be estimated very carefully in order to avoid numerical diffusion. First, it must satisfy the Courant-Friedrichs-Lewy condition (CFL) [18] in a manner as to guarantee the stability of the numerical scheme:

$$
\Delta t<\frac{\Delta x}{\mu E}
$$

where $\mu E$ is the carriers velocity estimated in each cell. Second, the time step $\Delta t$ must be less than the quickest phenomena occurring in each cell, only recombination processes in our case. Finally, $\Delta t$ must be defined in order to avoid numerical instability due to injection processes. For this latter constrain, an upper limit of $\Delta t$ of $20 \mu$ s has been found convenient.

Equations (7), (8) and (9) are resolved using the finite volume method. This method of finite volumes, which uses a discretization of the domain, works with volumes of control and makes it possible to transform the partial differential equations into continuous linear equations in the domain of calculation. This method has been chosen for its direct consistency with the conservation principle and for its relative simplicity.

\section{Phase-resolved EL measurements}

An experimental arrangement has been developed to measure EL emission from polymeric samples in a uniform field configuration at room temperature under AC stresses, Figure 3. This arrangement has been used previously for a range of measurements on various dielectric materials [19]. Measurements were 
undertaken on a $100 \mu \mathrm{m}$ thick low density polyethylene (LDPE) film metallized with semi-transparent gold layers ( $20 \mathrm{~nm}$ thick) in order to allow a uniform electrical field to be applied. The setup utilizes a Peltier-cooled electron multiplying charge coupled device (EMCCD) camera as a detection unit, allowing imaging of the emission. In addition to imaging of EL, the system can be reconfigured to determine the phase angle relationship of the emission with respect to the applied voltage. The camera is triggered at the zero crossing of the positive half-cycle to allow the synchronization of EL with the alternating electric field.

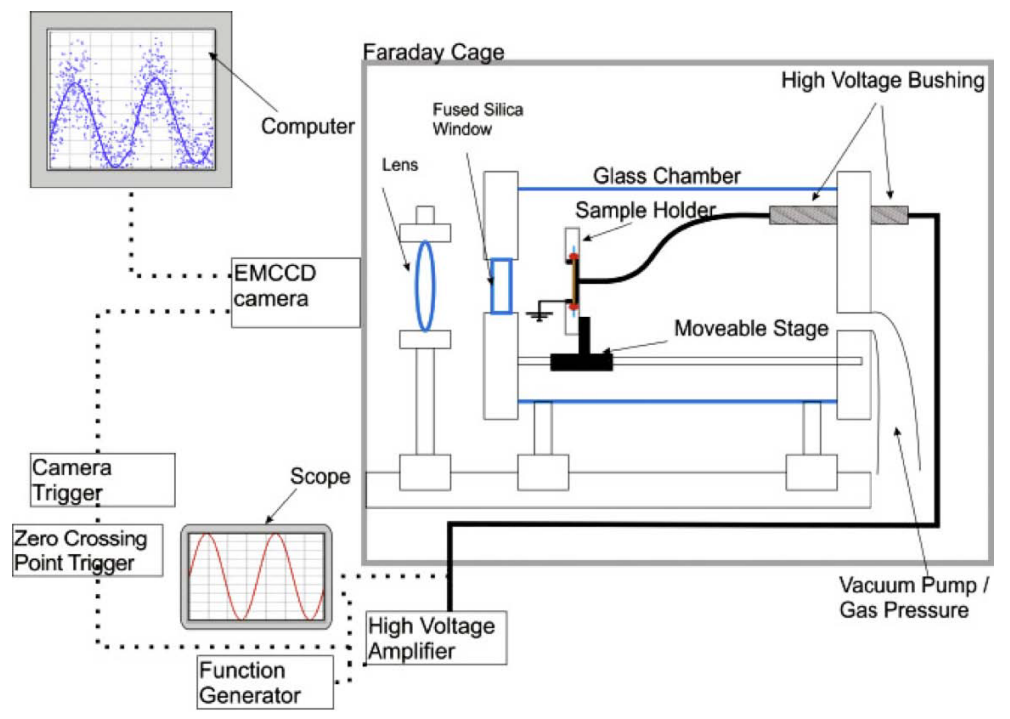

Figure 3. The experimental setup for measuring phase-resolved EL

After positioning the sample, the glass chamber was sealed and air evacuated to remove moisture using a vacuum pump until a pressure around 2 mbar was achieved. The chamber was then pressurized with $1 \mathrm{bar}$ of dry nitrogen in order to avoid corona discharges within the chamber due to the application of high voltages. The EMCCD camera was allowed to cool down to its normal operating temperature of $200 \mathrm{~K}$. The sample under test was subjected to various AC stresses by controlling the signal fed into a power amplifier. The phase resolved measurement consists of 1000 measurements of $2.168 \mathrm{~ms}$ each. Every value could be equated to a point on the applied field and measurements are repeated and averaged 100 times to improve signal to noise ratio. Experiments were performed with different waveforms, such as periodic sinusoidal, square and triangular waveforms and using a constant frequency of $50 \mathrm{~Hz}$. In all the following figures, the voltage has the polarity of the electrode furthest from the lens, so called "HV electrode" in Figure 1.

\section{Results}

The main goal in simulation is to reproduce the experimental EL phenomena for understanding the mechanisms at play. Simulations were performed in situations where a $6 \mathrm{kV}$ peak voltage was applied across the polymer film with different $50 \mathrm{~Hz}$ waveforms. Simulations were performed for a stressing time of $5 \mathrm{~s}$, using symmetrical model parameters for electrons and holes. In the following figures, data plotted correspond to the last period of the AC stress. All simulated EL intensity data, obtained from Eq. 6, have been multiplied by the same coefficient in order to have both experimental and simulation plots in the same figure for the sake of comparison. In the following, for convenience we assimilate recombination rates obtained from simulation and EL intensity obtained from the experiment. It must be realized that there is a gap between these two quantities, first because every recombination event does not necessarily produce photon emission (luminescence yield), second because light emission does not necessarily originate from recombination processes, as discussed in the last section of the paper. The optimized set of 
model parameters giving the best fit to experimental data is shown in Table 1 and will be commented on later.

With these parameters, the total traps density is about $7 \times 10^{27} \mathrm{~m}^{-3}$. Figure 4 shows the mobility as a function of the charge density for different values of the electric field. It appears that the mobility is strongly dependent on trap filling but not on the field itself. Mobility increases drastically for charge density $>10^{+6} \mathrm{C}^{-\mathrm{m}^{-3}}$. So high charge densities are actually obtained in the simulations in regions adjacent to the electrodes where charges accumulate, producing significant mobility enhancement.

Table 1: Set of optimized parameters

\begin{tabular}{|c|c|c|}
\hline Symbol & Value & Units \\
\hline $\begin{array}{c}\text { injection coefficients } \\
\alpha \\
\beta\end{array}$ & $\begin{array}{l}3 \times 10^{-3} \\
7 \times 10^{-8}\end{array}$ & $\begin{array}{l}\text { A. } \mathrm{m}^{-2} \\
\mathrm{~m} \cdot \mathrm{V}^{-1}\end{array}$ \\
\hline $\begin{array}{c}\text { recombination coefficients } \\
S_{e} \text { (electrons) } \\
S_{h} \text { (holes) }\end{array}$ & $\begin{array}{l}10^{-2} \\
10^{-2}\end{array}$ & $\begin{array}{l}\mathrm{m}^{3} \mathrm{C}^{-1} \mathrm{~s}^{-1} \\
\mathrm{~m}^{3} \mathrm{C}^{-1} \mathrm{~s}^{-1}\end{array}$ \\
\hline $\begin{array}{c}\text { transport coefficients for } \\
\text { electrons } \\
\Delta_{\text {max,e }} \\
N_{e}^{\prime} \\
T_{0, e}\end{array}$ & $\begin{array}{l}0.67 \\
10^{47} \\
10^{4}\end{array}$ & $\begin{array}{c}\mathrm{eV} \\
\mathrm{m}^{-3} \cdot \mathrm{J}^{-1} \\
\mathrm{~K}\end{array}$ \\
\hline $\begin{array}{l}\text { transport coefficients for holes } \\
\qquad \Delta_{\max , \mathrm{h}} \\
N_{h}^{\prime} \\
T_{0, h}\end{array}$ & $\begin{array}{l}0.67 \\
10^{47} \\
10^{4}\end{array}$ & $\begin{array}{c}\mathrm{eV} \\
\mathrm{m}^{-3} \mathrm{~J}^{-1} \\
\mathrm{~K}\end{array}$ \\
\hline
\end{tabular}

As an example, for an applied crest field of $60 \mathrm{kV} \cdot \mathrm{mm}^{-1}$ and whatever the waveform (sinusoidal, triangular or square), mobility is about 3 times higher close to the electrodes than within the bulk (the corresponding density of trapped charge is of the order of $10^{7} \mathrm{C} \cdot \mathrm{m}^{-3}$; mobility reaches $6.10^{-16} \mathrm{~m}^{2} . \mathrm{V}^{-1} \cdot \mathrm{s}^{-1}$ adjacent to the electrodes and $2 \cdot 10^{-16} \mathrm{~m}^{2} \cdot \mathrm{V}^{-1} \cdot \mathrm{s}^{-1}$ in the bulk).

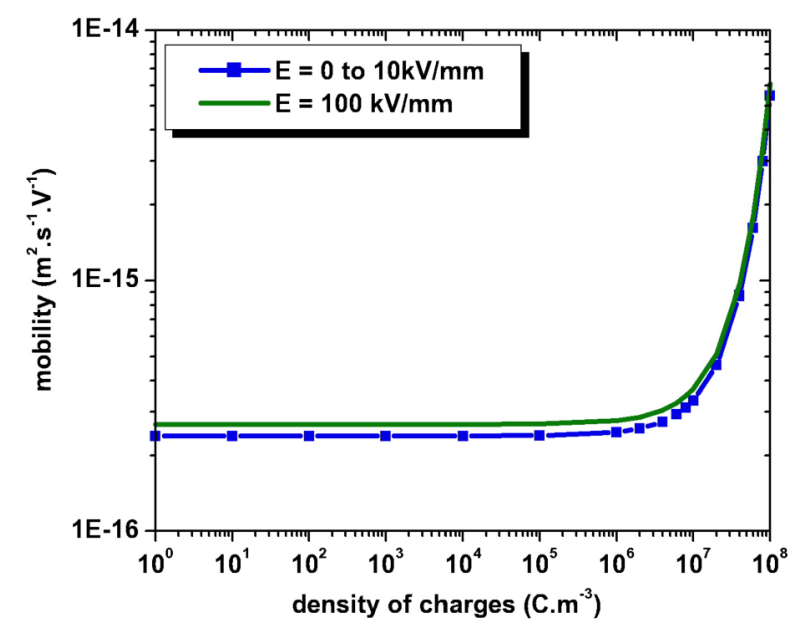

Figure 4. Mobility function of electric field and charge density using parameters of Table 1

\subsection{Sinusoidal waveform}

Figure 5 compares experimental and simulated EL patterns for a $50 \mathrm{~Hz}$ sinusoidal waveform. Quite a good fit is obtained for the entire period of AC stress. Two peaks in EL intensity are observed, as usual in this kind of measurement. They are seen in each AC cycle, the EL peaks being in advance relative to the 
voltage maxima by an angle $\varphi_{1}$ for the positive half cycle and $\varphi_{2}$ for the negative half cycle as specified in Table 2. Small differences are seen in the values of these phase shifts between experiments and simulation, especially for the negative half cycle.

Table 2: Phase shift between EL peaks and max. of voltage for both half cycles.

\begin{tabular}{lll}
\hline & $\varphi_{1}$ & $\varphi_{2}$ \\
\hline EL measurement & $38^{\circ}$ & $21.8^{\circ}$ \\
\hline EL simulation & $32.4^{\circ}$ & $32.4^{\circ}$ \\
\hline
\end{tabular}

Figure 6 shows the simulated electric field and charge density along the last simulated cycle. Charge densities being represented correspond to the positive, negative, and net charge averaged within the first $10 \mathrm{~nm}$ from the reference electrode: beyond this value, the charge density becomes insignificant and is neglected. It should be realized that the net charge density is oscillating from positive to negative crossing the zero charge line but this is due to the mutual compensation of a high density of charges of opposite sign: trapped charge as high as $10^{6} \mathrm{C} . \mathrm{m}^{-3}$ are predicted by this model.

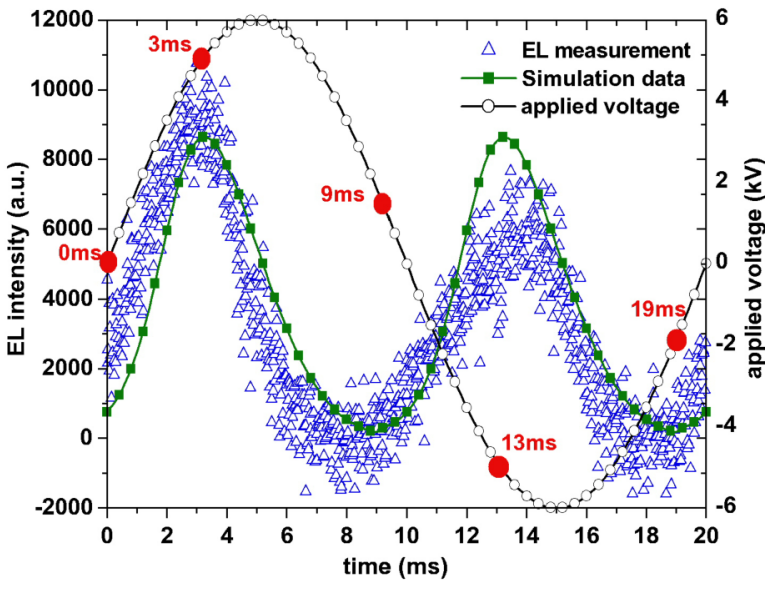

Figure 5. Comparison between EL measurement and simulation under sinusoidal waveform

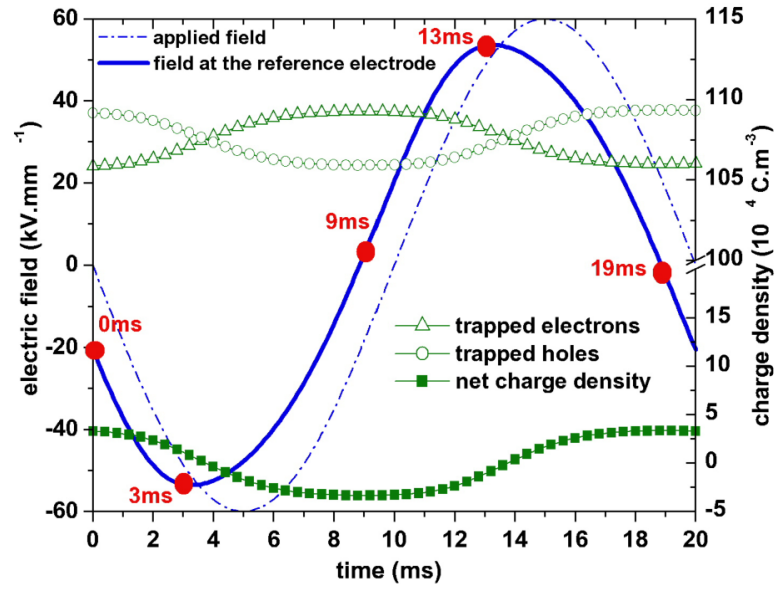

Figure 6. Electric field and density of charge vs. time for sinusoidal waveform

The spatial distribution of the net charge density and electric field close to the reference electrode are shown in Figure 7 at different times (specified by black circles in Figure 8) of the AC cycle. Figure 8 shows the electrode current for electrons and holes and electric field for one cycle. Analysis of both figures allows several comments to be made.

At $t=0$ (zero applied voltage), electric field at the reference electrode is negative (because the net density of charge is positive due to the previous accumulated positive charge within the positive half cycle) and hence electrons begin to be injected and holes begin to be extracted. Injected electrons are then trapped and some of them recombine with trapped holes: hence, the recombination between injected electrons and trapped holes increases in time, $c f$. Figure 5 . At $t=3 \mathrm{~ms}$, electric field at the cathode, recombination rate, injection current for electrons and extraction current for holes are practically at their maximum. The net density of charge begins to be negative (in the first cell, charge is negative, $c f$. Fig.7) and the electric field at the cathode reaches progressively the applied field. This appears prior to the maximum of the applied field. Between $t=3 \mathrm{~ms}$ and $t=9 \mathrm{~ms}$, negative carriers are majority carriers in all the cells close to the cathode and this homocharge induces a lowering of the applied field. In this time interval, the recombination rate between injected electrons and trapped holes decreases, i.e. the EL intensity decreases. Between $t=9 \mathrm{~ms}$ and $t=13 \mathrm{~ms}$, the electric field at the reference electrode is positive, positive carriers are injected but the net charge density remains negative, acting as an heterocharge that enhances the electric field at the reference electrode relative to the applied field. The recombination between injected 
holes and trapped electrons begins to increase. At $t=13 \mathrm{~ms}$, the electric field at the reference electrode, recombination rate, injection current for holes and extraction current for electrons are practically at their maximum. The net density of charge begins to be positive (in the first cell, charge is positive) and the electric field at the electrode reaches progressively the applied field. As previously, this feature appears before the maximum of the applied field. Between $t=13 \mathrm{~ms}$ and $t=19 \mathrm{~ms}$, positive carriers are still injected (positive electric field at the electrode) and reduce the electrode field. Injection current for the holes decreases and hence the recombination between injected holes and trapped electrons decreases.

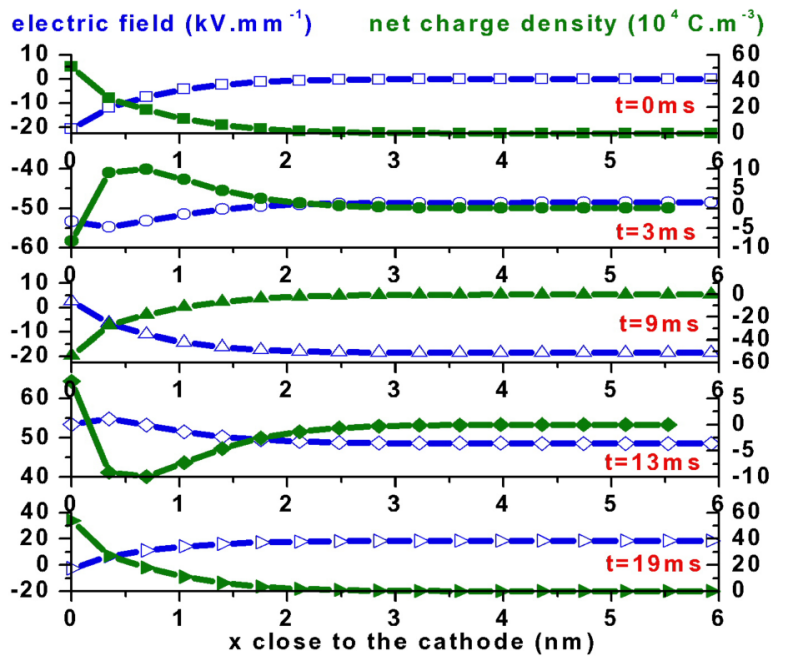

Figure 7. Electric field (void symbol) and net charge density (full symbol) vs. depth for sinusoidal waveform

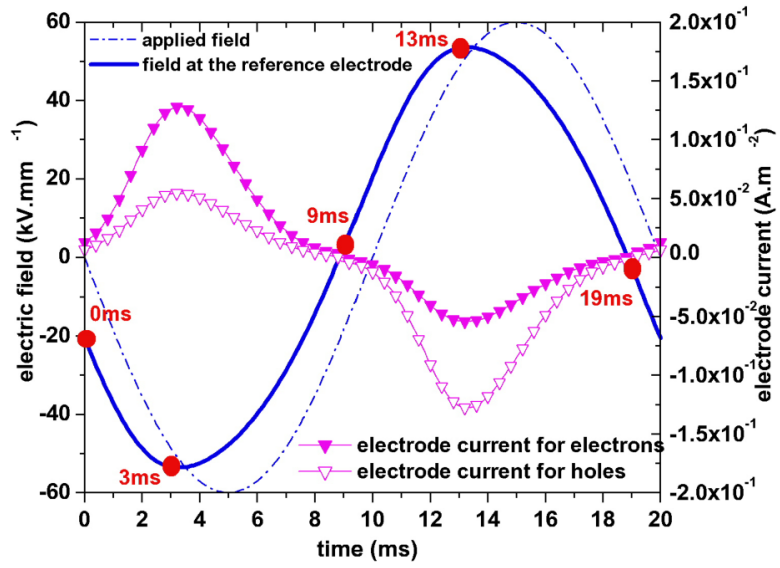

Figure 8. Electric field and electrode current vs. time for sinusoidal waveform

\subsection{Triangular waveform}

The EL emission for triangular waveform is shown in Figure 9. Again, the fit between experiment and simulation is very good: EL intensity peaks at voltage maxima, increases relatively slowly in ramping up the voltage, and decreases much more rapidly in ramping down.

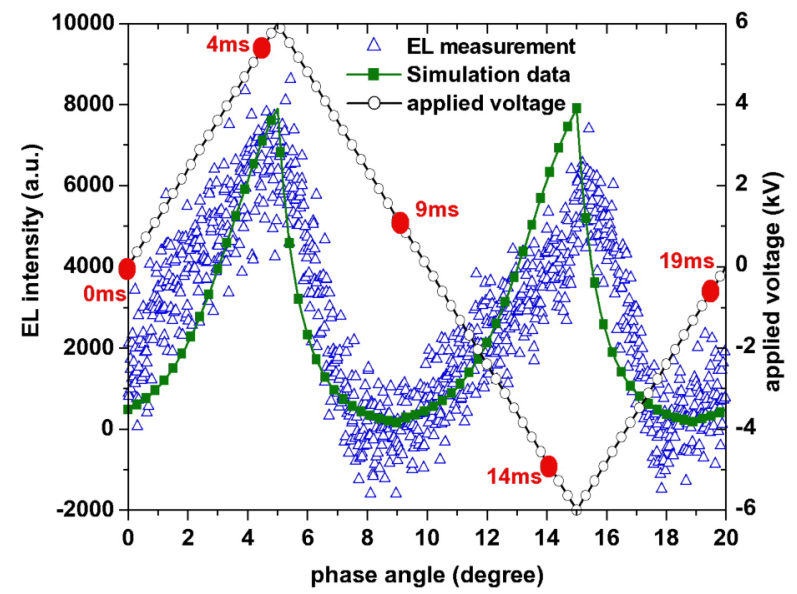

Figure 9. Comparison between EL measurement and simulation under triangular waveform

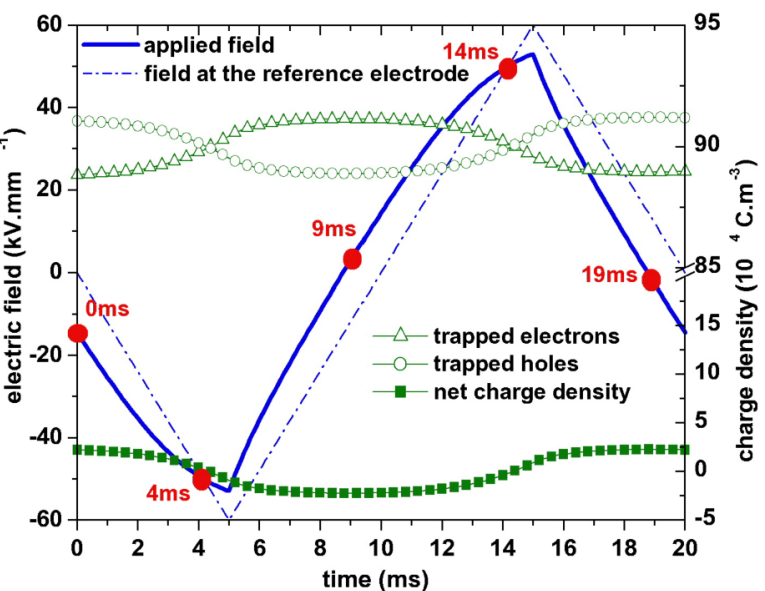

Figure 10. Electric field and density of charge vs. time for triangular waveform

Figure 10 shows the net charge, trapped electron and trapped hole densities along the last simulated cycle. Again, these values have been obtained by averaging the charge within $10 \mathrm{~nm}$ from the electrode. The charge density is of the same order as that simulated in the sinusoidal case and hence induces a significant 
deformation of the electric field at the electrode that becomes lower than the applied field $1 \mathrm{~ms}$ before the peak in applied voltage. This strong deformation is not enough to shift the peak of the electric field at the electrode relative to the applied voltage. This is a striking difference when compared to the sinusoidal case that will be discussed later.

The spatial distribution for the net charge density and electric field close to the reference electrode are shown in Figure 11 at different times (specified by black circles in Figure 12) during the last simulated cycle. As already pointed out, a charge value below $1 \mathrm{C} . \mathrm{m}^{-3}$ is not taken into account. Figure 12 shows the electron and hole currents and the electric field at the reference electrode over one cycle.

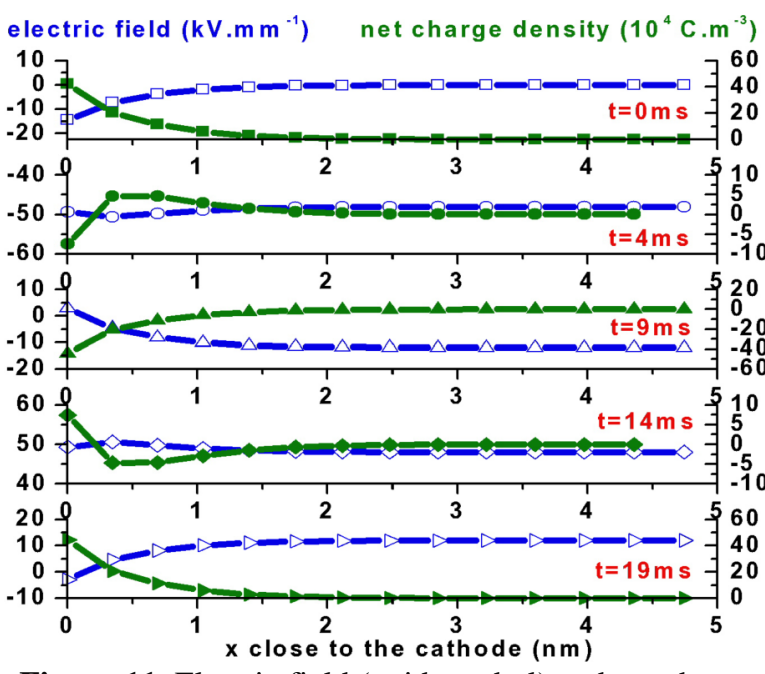

Figure 11. Electric field (void symbol) and net charge density (full symbol) vs. depth for triangular waveform

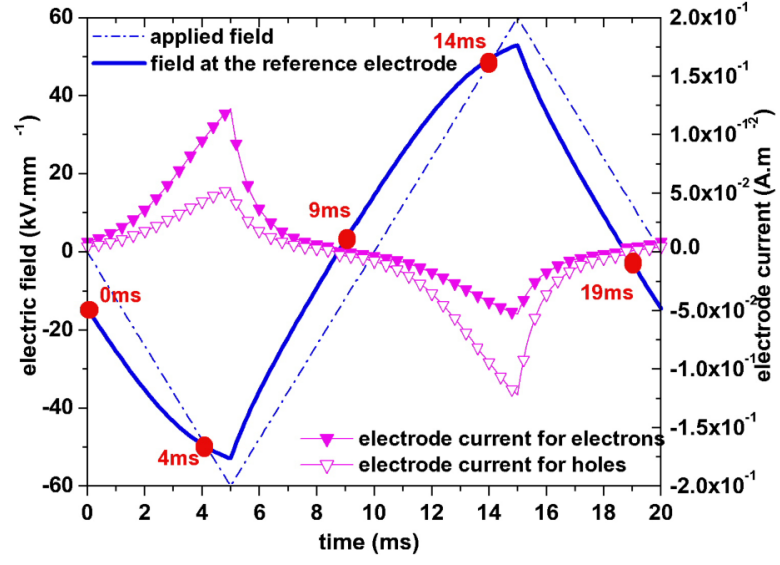

Figure 12. Electric field and electrode current vs. time for triangular waveform

Space charge behaves qualitatively in a similar way as in the case of sinusoidal applied waveform even though the space charge penetration depth over $5 \mathrm{~s}$ is smaller (less than $5 \mathrm{~nm}$ in triangular waveform). Between $t=0 \mathrm{~ms}$ and $t=4 \mathrm{~ms}$, electrons are injected and are either trapped or recombined with the previously trapped holes. At the same time some holes are extracted. In this time interval, heterocharges are observed and the electric field at the electrode increases. Between $t=4 \mathrm{~ms}$ and $t=9 \mathrm{~ms}$, negative carriers are dominant close to the electrode (acting as homocharges). After reaching its maximum at $t=5 \mathrm{~ms}$, recombination between injected electrons and trapped holes decreases. The same features are observed for the second half cycle when holes are injected and electrons extracted.

\subsection{Square waveform}

EL patterns under a square waveform are shown in Figure 13. The characteristics of the square voltage pulses are such that the rise and fall time of the voltage are $1 \mathrm{~ms}$. This feature has been introduced in the simulation as well.

As shown in Figure 13, EL emission starts at voltage polarity inversion and peaks at voltage maxima. Indeed, the rapid change from positive to negative applied voltage (or vice versa) causes a massive charge injection into the dielectric, leading to an increase in the number of recombination events. In simulation trials, hypothesizing an abrupt change in the voltage from $-\mathrm{V}_{\max }$ to $+\mathrm{V}_{\max }$, i.e. $\mathrm{dV} / \mathrm{dt}$ is 'infinite', leads to a peak of EL at polarity reversal followed by a monotonous decrease, underlining the importance of $\mathrm{dV} / \mathrm{dt}$ value.

Figure 14 shows the net charge along with trapped electrons and trapped holes densities during the last simulated cycle, averaging data over $10 \mathrm{~nm}$ at the reference electrode. Apart from the fact that again with this voltage waveform, the net charge density results from an unbalanced situation between much higher densities of charges of both polarities, the striking result is that the electrode field at voltage maxima remains higher than the applied field for about $1 \mathrm{~ms}$. This was not observed in sinusoidal and triangular 
excitation where the electrode field at voltage maxima was reduced by the injected space charge. This is obviously to be put in relation with the balance between the amount of charges provided by injection and its reduction through extraction/recombination. The dynamic of the excitation (i.e. $\mathrm{dV} / \mathrm{dt}$ ) seems to be the controlling factor; this will be discussed later on.

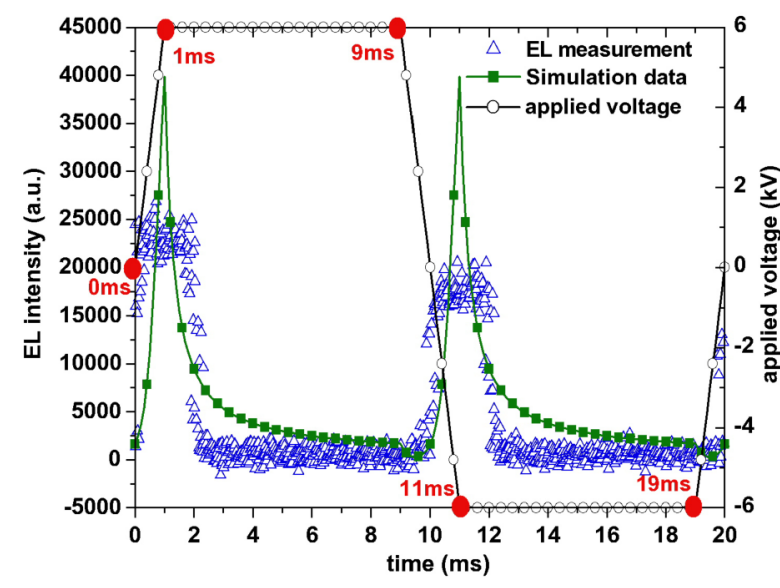

Figure 13. Comparison between EL Measurement and EL Simulation under square waveform

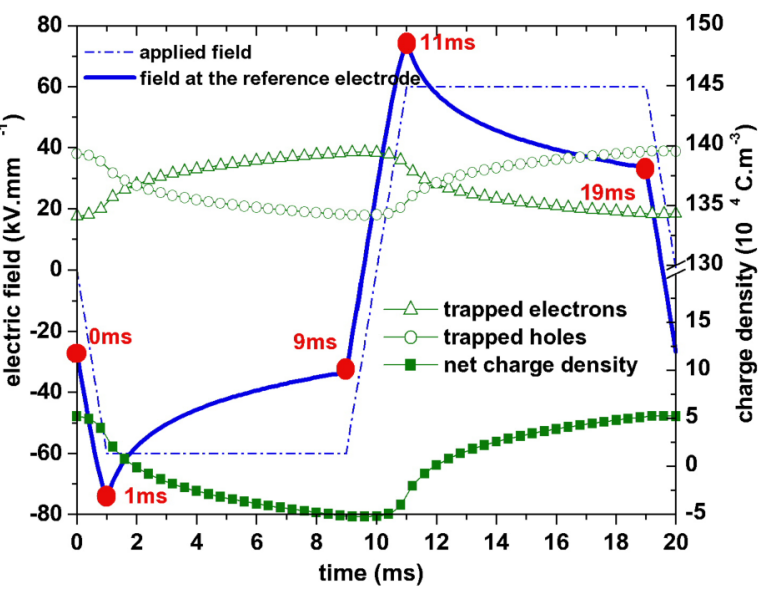

Figure 14. Electric field and density of charge vs. time for square waveform

The spatial distribution for the net charge density and the electric field close to the reference electrode is shown in Figure 15 at different times (specified by black circles in Figure 16) during the last simulated cycle. Figure 16 shows the injection current for electrons and holes as well as the electric field for one cycle.

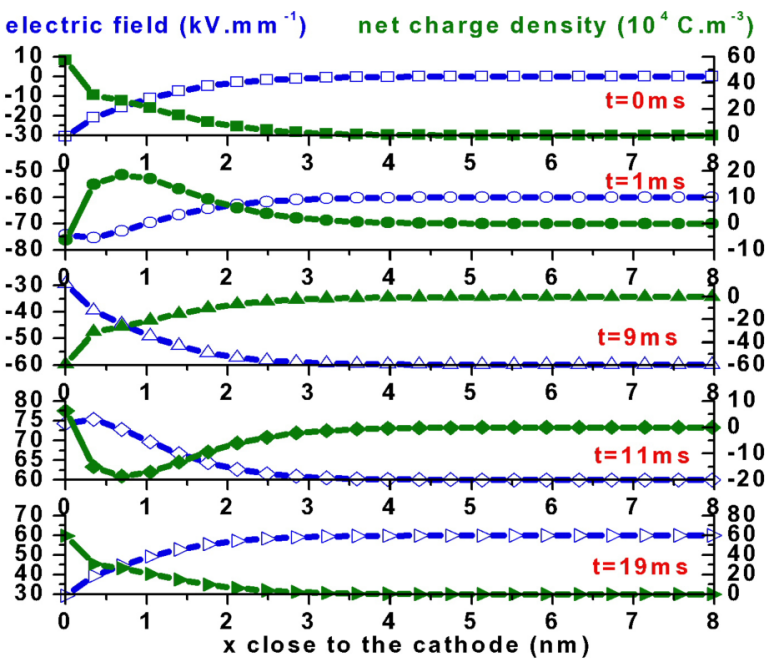

Figure 15. Electric field (void symbol) and net charge density (full symbol) vs. thickness for square waveform

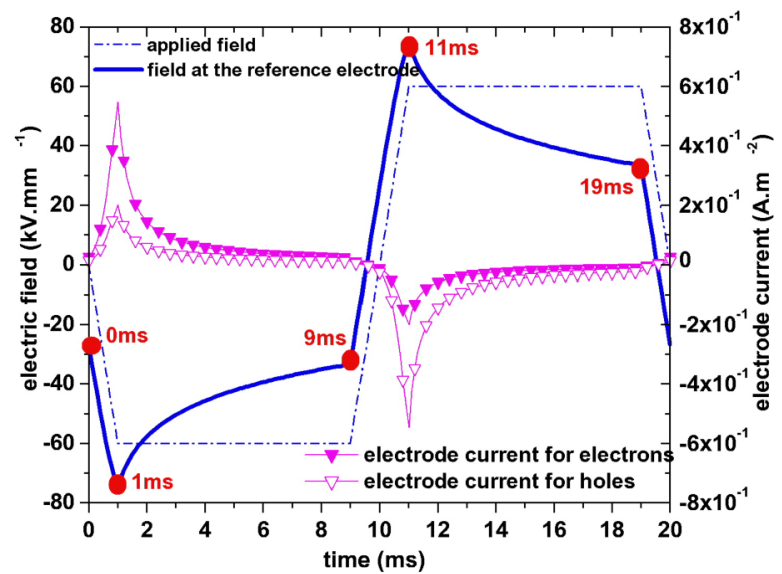

Figure 16. Electric field and electrode current vs. time for square waveform

Between $\mathrm{t}=0 \mathrm{~ms}$ and $\mathrm{t}=1 \mathrm{~ms}$, heterocharges are seen up to $3 \mathrm{~nm}$ away from the cathode. A part of this charge is extracted or recombined by the injected carriers as depicted by the decrease of the positive charge density (specially close to the cathode) but the positive charge is still dominating at $t=1 \mathrm{~ms}$. This explains why the electric field is enhanced (up to $75 \mathrm{kV} / \mathrm{mm}$ ) contrary to what was observed for sinusoidal and triangular excitation waveforms. With ongoing negative charge injection, the net charge appears negative at $\mathrm{t}=9 \mathrm{~ms}$ and the electrode field is reduced below the applied field (from $2 \mathrm{~ms}$ onwards). From 
$\mathrm{t}=9 \mathrm{~ms}$ to $\mathrm{t}=11 \mathrm{~ms}$, voltage linearly increases from $-\mathrm{V}_{\max }$ to $+\mathrm{V}_{\max }$ and the electrode field is enhanced due to a negative net charge up to $\mathrm{t}=12 \mathrm{~ms}$. This is shown in Figure 15 where the dominating charge is still a negative charge until $\mathrm{t}=12 \mathrm{~ms}$ (not shown in Figure 15). From $\mathrm{t}=12 \mathrm{~ms}$ to $\mathrm{t}=19 \mathrm{~ms}$, positive injection takes the lead and the net charge is positive leading to the lowering of the electrode (anode) field. This is also clearly depicted in Figure 14.

\section{Discussion}

A first general observation concerns the nice fit between experiments and simulations. For the three excitation waveforms, the model was able to reproduce closely the experimental behaviour. The luminescence peaks were reproduced and their origin can be understood on the basis of the charge injection and charge distribution at different times. Note that all the simulation results featuring charge penetration into the dielectric were presented in the vicinity of one electrode, alternatively behaving as cathode and anode depending on the voltage polarity. Because the same transport parameters were used in the model for positive and negative charge carriers, the situation at the other electrode is purely identical (with appropriate polarity) for charge penetration depth, charge density, electric field distribution, etc.

A general feature of the results is that the EL peaks when injection current is at a maximum, the latter being always correlated with a maximum in the recombination rate. This also corresponds to a maximum in the local field at the injecting electrode. A first consequence is that it is impossible experimentally to differentiate between hot electron excitation vs. recombination on the basis of a phase shift between EL and current. As discussed by Zeller [20] hot electron-induced luminescence and recombination-induced luminescence both scale with current. Another implication concerns the nature of the EL when observed on metallized samples of the type used in this study. Because injection current can also excite surface plasmons on the electrode surface [21], and because these collective electronic excitations are able to decay radiatively, the only way to differentiate between the latter and EL from the dielectric is to avoid surface plasmons relaxation in the visible range (this can be done by using metal electrode other than gold -ITO (Indium-Tin Oxide) for example [22]). An alternative way is to analyze the emission spectrum in specific regions characteristic of the polymer and/or by filtering out the light from relaxation of surface plasmons [21]. Another interesting point coming from the modelling is the value of the injection current at peak maxima, which is of the order of $10^{-1} \mathrm{~A} \cdot \mathrm{m}^{-2}$.

It is interesting to note some differences in the EL phenomenology considering the three different types of excitation waveforms. Under sinusoidal excitation, the field at the injecting electrode is lowered in comparison to the applied field before voltage maximum and the EL peaks are in advance relative to the applied field. In triangular excitation, the field at the injecting electrode is also reduced before voltage maximum but the EL peaks are in phase with the applied field. Using the square excitation, the field is reduced below the applied field after the voltage reaches its maximum and is higher than the applied field during voltage rising up (and for $1 \mathrm{~ms}$ under constant stress, decreasing after this time). This is a consequence of the charge distribution in the vicinity of the electrode as described in the previous section. For square excitation waveform, the voltage rising rate is $6 \mathrm{kV} / \mathrm{ms}$, much higher than for the triangular waveform $(1 \mathrm{kV} / \mathrm{ms})$. The consequence is that the time the sample is subjected to the field up to the voltage maximum is shorter for larger $\mathrm{dV} / \mathrm{dt}$. It follows the recombination/extraction of the trapped heterocharge tends to be incomplete and the amount of remaining heterocharge controls the electric field. This is a strong point of the model to describe dynamical equilibrium between charge injection, transport and recombination.

Other points of interest concern the density and penetration depth of the charge. Typical charge density in the model is between $10^{+4} \mathrm{C} . \mathrm{m}^{-3}$ and $10^{+7} \mathrm{C} . \mathrm{m}^{-3}$, i.e. two to four orders of magnitude higher than the charge density reported in typical experimental work. Moreover, it seems to be independent of the shape of the excitation. If the charge density values for a given type of charge appears high $\left(10^{+6} \mathrm{C}_{\mathrm{m}} \mathrm{m}^{-3}\right)$, one has to realize that the net charge density is 20 times lower and results from an unbalanced situation between positive and negative densities. What is experimentally measured with any space charge detection technique is always the net charge and one has to realize that a given net charge density can result from the superposition of charge distribution of both polarities with a much higher density. Orders of magnitude 
can be derived from a recent paper [23] where time-resolved space charge distribution has been measured in polyethylene under $\mathrm{AC}$ voltage up to a frequency of $10 \mathrm{~Hz}$. Charge injection in every half cycle was evidenced at low frequency (up to $0.1 \mathrm{~Hz}$ ) and merely detectable above, even if the range of field (30 $\mathrm{kV} / \mathrm{mm}$ ) was lower than in the current study. Temperature effects were also evidenced (charge injection evidenced up to $10 \mathrm{~Hz}$ ) as well as a negative charging of the material for long stressing times, denoting a dissymmetry in transport parameters for positive and negative charge carriers. Simulation and experimental results can be reconciled if one considers that the actual space charge distribution controlling the EL emission concerns a layer of only few $\mathrm{nm}$ where the space charge has a high density. Considering the usual spatial resolution of the detection technique - at best of the order of $10 \mu \mathrm{m}$, one can deduce that a net charge density of $10^{+4} \mathrm{C} . \mathrm{m}^{-3}$ established over a layer of $10 \mathrm{~nm}$ (overestimation of the simulation result) would be detected as a space charge region of $10 \mathrm{C} . \mathrm{m}^{-3}$ in density. Therefore it is not surprising that a thin layer of charges cannot be easily identified experimentally. Another point of interest concerns the negative charging of the material observed for long stressing times. This indicates non symmetrical transport parameters for both types of charges, which has not been attempted so far in the current version of the modelling. The other difficulty here is that long stressing times are necessary (order of hours), and that the simulation is computationally intensive (this is the reason why we have limited the stressing time to $5 \mathrm{~s}$ in the current results).

Charge penetration depth spans from $5 \mathrm{~nm}$ to $8 \mathrm{~nm}$ depending on the excitation waveform. Penetration depth with square excitation is higher than with sinusoidal excitation, that is itself higher than with triangular excitation, consistently with the time allowed for charge transport under constant stress: in fact, the field reduction factor, i.e. the ratio between the electrode field and the applied field is all the larger that the time for charge accumulation is long: about $50 \%$ at the end of the voltage plateau in square waveform vs. about $10 \%$ at peak voltage for sinusoidal or triangular waveform.

Regarding EL intensity, from the experimental results it is clear that the EL measured during the positive voltage half cycle is about $30 \%$ higher than that during the negative half cycle for all excitation waveforms. When the electrode facing the collecting lens is at a positive voltage, the EL at the anode is not affected by the optical transmission of the polymer contrary to the EL from the cathode (absorption of the semi-transparent gold electrode affects the recorded signal at the both electrodes the same way). When the polarity is changed, the EL from the anode is now affected by the optical absorption. One should therefore get the same EL intensity in every half cycle if the processes leading to light emission were the same at cathode and anode. This is indeed observed in simulation were the same transport parameters have been adopted for positive and negative charge carriers but not in experiments, denoting some asymmetry in the charge behaviour. This is in fact what could be expected from the experimental results in [24] that show a negative charging of the polymer for long stressing time under AC. This suggests an unbalanced situation between negative carrier production/annihilation, leading to an effective transport of negative charge. If this is so, more EL should be generated upon positive injection explaining the experimental results. However, other factors could also play a role like for example the difference in nature of the recombination centres for positive and negative charges leading to different emission efficiency for the same density of excited species. Further work in the modelling of real-time space charge distribution under AC using the actual model is currently being considered. Considering the EL intensity per se, one can note that it is lower for sinusoidal and triangular applied voltages when compared to the square voltage: the ratio between EL peaks for square and triangular or sinusoidal waveforms is about 3 when taking readings from the experimental results. The same trend holds in simulation but the simulated EL for the square voltage does not follow perfectly the experimental behaviour at the peak. One general remark is that the EL intensity scales with the injection current in every case and the large difference between sinusoidal/triangular and the square excitation comes from the large difference in injection current. The misfit between simulated and measured EL at the peak for the square excitation comes from experimental averaging of the EL during 2 ms which naturally smeared out any sharp maximum in the EL.

One of the fundamental questions raised by our results is the physical soundness of the model and the meaning of the optimized parameters. As recalled in Table 1, model parameters pertain to the injection law and the exponential trap distribution. As regards the injection law, parameters $\alpha$ and $\beta$ have been 
initialized from those used in [19] and optimized. For the trap distribution, one way to proceed for parameterizing is to consider literature data on trap density estimates. Quirke et al. [24, 25] have developed a molecular simulation aimed at estimating trap depth associated to given chemicals in polyethylene, along with physical traps associated with disorder in chain conformation. It has already been shown [13] that the exponential distribution reasonably accounts for these molecular simulation results. The parameters used in this study have been optimized from those given in [13].

\section{Conclusion}

A transport model accounting for the EL phenomena by recombination of charge carriers in LDPE under AC stress has been proposed and tested by comparing simulated and experimental results. The model incorporates injection/extraction as well as transport and recombination of charges. Charges are transported according to a thermally-assisted hopping mobility between traps having an exponential energy distribution in such a way that the mobility is modulated by the filling of traps. Transport parameters are the same for positive and negative charge carriers. A good correlation between measurements and simulation has been obtained regarding the time-dependence of EL intensity for different types of applied voltage waveforms, being $50 \mathrm{~Hz}$ sinusoidal, triangular and square voltage.

Interpretation of the EL dependency on voltage waveform has been discussed considering penetration depth and density of charge carriers derived from the simulation. The salient feature of the finding is that the density of charge carriers needed to explain the EL emission is higher by four orders of magnitude than the typical density measured with the space charge measurement techniques. This can be reconciled when taking into account the thickness of the dielectric layer where charges are trapped which is less than $10 \mathrm{~nm}$, leading to a charge density of the order of $1 \mathrm{C}^{-3} \mathrm{~m}^{-3}$ when averaged over a thickness typical of the spatial resolution of space charge measurement techniques. The simulation results allow inferring such behaviour only because the spatial discretization of the problem is very tight near electrodes with computation cells of $0.4 \mathrm{~nm}$ each within a distance of $3.5 \mathrm{~nm}$ from the electrode.

If the actual model seems to give a correct description of the interfacial processes leading to light emission upon charge recombination, the challenge will be to demonstrate its ability to take into account charge transport under AC and DC stresses. Because the transport properties within the surface layer of the polymer are unlikely to be the same as in the bulk, describing charge transport by using a unique distribution of traps seems unlikely. This will have to be incorporated in further work but investigation of the EL under time-varying voltage could provide a way to probe the electric properties at the interfaces.

\section{References}

[1] Fleming RJ 2005 Space charge profile measurement techniques: recent advances and future directions IEEE Trans. Electr. Insul. 12 967-78

[2] Meunier M and Quirke N 2000 Molecular modeling of electron trapping in polymer insulators $J$. Chem. Phys.113, 369-76.

[3] Sanche L 1993 Electronic aging and related electron interactions in thin-film dielectrics IEEE Trans. Electr. Insul. 28, 789-819.

[4] Dissado L A, Mazzanti G and Montanari G C 2001 Elemental strain and trapped space charge in thermoelectrical aging of insulating materials. Part I: elemental strain under thermo-electrical-mechanical stress 2001 IEEE Trans. Dielectr. Electr. Insul. 8 959-965.

[5] Le Roy S, Teyssedre G and Laurent C 2005 Charge transport and dissipative processes in insulating polymers: joining experiments and model IEEE Trans. Dielectr. Electr. Insul. 12, 644-54.

[6] Shimizu N, Kosaki M and Horii K 1977 Space charge effect on local electric breakdown of polyethylene at 77K J. Appl. Phys. 48 2191-95.

[7] Hartman W A and Armstrong H L 1967 Electroluminescence in organic polymers J. Appl. Phys. 38 2393-95. 
[8] Laurent C, Massines F and Mayoux C 1997 Optical emissions due to space charge effects in electrically stressed insulating polymers IEEE Trans. Dielectr. Electr. Insul. 4 585-603.

[9] Alison J M, Champion J V, Dodd S J and Stevens GC 1995 Dynamic Bipolar Charge Recombination Model for Electroluminescence in Polymer Based Insulation during Electrical Tree Initiation J. Phys. D: Appl. Phys. 28 1693-701.

[10] Champion J V, Dodd S J and Stevens G C 1994 Long Term Light Emission Measurement and Imaging during the Early Stages of Electrical Breakdown in Epoxy Resin J. Phys. D: Appl. Phys. 27 604610.

[11] Mohd Ariffin A, Mat Tajudin N, Sulaiman S, Md Thayoob Y H, and Lewin P L 2010 Comparing Simulation Results and Experimental Measurements of Electroluminescence Phenomenon in Dielectric Materials Proc. IEEE 2010 International Symposium on Electrical Insulation, pp. 1-5, 6-9 June 2010, San Diego, Californie, USA.

[12] Le Roy S, Teyssedre G, Laurent C, Montanari G C and Palmieri F 2006 Description of charge transport in polyethylene using a fluid model with a constant mobility : fitting model and experiments $J$. Phys. D: Appl. Phys., 39 1427-36.

[13] Boufayed F, Teyssedre G, Laurent C, Le Roy S, Dissado L A, Segur P and Montanari G C 2006 Models of bipolar charge transport in polyethylene J. Appl. Phys. 100104105.

[14] Mott N F and Davis E A, Electronic Processes in Non-Crystalline Materials, 2nd Ed., Clarendon Press Ed., Oxford, 1979.

[15] Hare R W, "Modelling space charge in solid dielectrics" Ph.D. Thesis, University of Bristol, 1993.

[16] Le Roy S, Segur P, Teyssedre G and Laurent C 2004 Description of bipolar charge transport in polyethylene using a fluid model with a constant mobility: model prediction J. Phys. D: Appl. Phys., 37 298-305.

[17] Teyssedre G, Tardieu G, Mary D and Laurent C 2001 AC and DC Electroluminescence in Insulating Polymers and its Implication for Electrical Ageing J. Phys. D: Appl. Phys 34, 2220-9.

[18] Press W H, Flannery B P, Teukolsky S A and Vetterling W T, Numerical Recipes, Cambridge University Press, Cambridge, Chap. 17, 1986.

[19] Cariou-Saintemarie N, Davies A E and Head J G, "Electroluminescence Measurements in Polyethylene Insulation" International Conference on Dielectric Materials, Measurements and Applications, 2002, pp. 430-3.

[20] Zeller H R Non insulating properties of insulating materials 1991 Annual Report Conference on Electrical Insulation and Dielectric Phenomena 19-47.

[21] Teyssedre G, Cissé L, Mary D and Laurent C 1999 Identification of the electroluminescence components of polyethylene IEEE Trans. Dielectr. Electr. Insul. 6 11-9,

[22] Aubert E, Teyssedre G, Laurent C, Rowe S and Robiani S 2009 Electrically-active defects in silicafilled epoxy as revealed by light emission analysis J. Phys. D: Appl. Phys. 42 165501-01/11.

[23] Thomas C, Teyssedre G and Laurent C Space-charge dynamic in polyethylene : from dc to ac stress 2011 J. Phys. D: Appl. Phys. 44015401.

[24] Meunier M, Aslanides A and Quirke N 2001 Molecular modeling of electron traps in polymer insulators : chemical defects and impurities J. Chem. Phys. 115, 2876-81.

[25] Anta J A, Marcelli G, Meunier M and Quirke N 2002 Models of electron trapping and transport in polyethylene : current-voltage characteristics J. Appl. Phys. 92, 1002-8. 